\title{
Conformation-dependent recognition of HIV Gp120 by DARPins provides novel possibilities to develop distinct HIV entry inhibitors
}

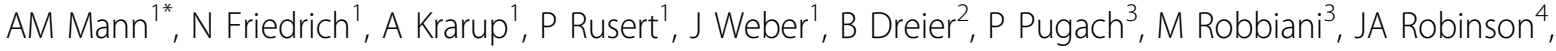 \\ A Pluckthun ${ }^{2}$, A Trkola $^{1}$
}

From AIDS Vaccine 2012

Boston, MA, USA. 9-12 September 2012

\section{Background}

Designed Ankyrin Repeat proteins (DARPins) are a novel type of binding protein scaffold designed as antibody alternative for biomedical applications. DARPins share many properties with antibodies, most noteworthy a high target specificity and affinity, but they differ from antibodies in size, structure and binding pattern and, importantly, favorable biophysical properties such as exceptional stability. This together with high-yield prokaryotic production renders DARPins promising candidates for microbicide development.

\section{Methods}

DARPin DNA libraries encoding either two or three internal ankyrin repeats were subjected to ribosome display selections and panned against recombinant gp120 proteins. Wild type, CD4 induced, V1V2 truncated and deglycosylated JR-FL gp120, as well as structural V3 loop mimetics were probed as target. Obtained DARPin clones were sequenced, mapped for reactivity with HIV gp120 by ELISA and probed for inhibitory activity using the TZMbl pseudotype virus inhibition assay.

\section{Results}

DARPin selection proved more successful when conformationally arrested targets were used for panning. Overall, gp120-specific DARPins recognizing a variety of epitopes including the CD4bs, CD4i and the V3 loop were obtained. Gp120 mutant binding analysis revealed that DARPin molecules depended to a higher degree on a structural conservation of the envelope protein than gp120 specific antibodies recognizing overlapping domains. Most noteworthy, V3 loop specific clones were selected, which unlike V3 loop antibodies, recognized the V3 loop in a conformation-dependent manner and thus do not efficiently bind linear V3 loop peptides. In contrast to V3loop directed antibodies these DARPins proved to bypass the envelope shielding by the V1V2 domain and thus were capable of neutralizing TIER-2 viruses.

\section{Conclusion}

Gp120 proved a challenging target for selection of DARPin binders against gp120. Nonetheless, by combining different selection strategies we were able to derive a variety of gp120-specific DARPin molecules, including some with unique HIV entry blocking activity.

\section{Author details}

${ }^{1}$ University of Zurich, Zurich, Switzerland. ${ }^{2}$ Institute of Biochemistry,

University of Zurich, Zurich, Switzerland. ${ }^{3}$ Center for Biomedical Research, Population Council, New York, NY, USA. ${ }^{4}$ Institute of Organic Chemistry, University of Zurich, Zurich, Switzerland.

Published: 13 September 2012

doi:10.1186/1742-4690-9-S2-P218

Cite this article as: Mann et al.: Conformation-dependent recognition of HIV Gp120 by DARPins provides novel possibilities to develop distinct HIV entry inhibitors. Retrovirology 2012 9(Suppl 2):P218.

'University of Zurich, Zurich, Switzerland

Full list of author information is available at the end of the article

(c) 2012 Mann et al; licensee BioMed Central Ltd. This is an Open Access article distributed under the terms of the Creative Commons 